\title{
PHYSICS AT THE FCC-ee
}

\author{
David d'Enterria \\ CERN, EP Department, 1211 Geneva, Switzerland
}

\begin{abstract}
The physics program accessible in $e^{+} e^{-}$collisions at the Future Circular Collider (FCC-ee) is summarized. The FCC-ee aims at collecting multi-ab ${ }^{-1}$ integrated luminosities in $e^{+} e^{-}$at $\sqrt{\mathrm{s}}=90,160,240$, and $350 \mathrm{GeV}$, yielding $10^{12} \mathrm{Z}$ bosons, $10^{8} \mathrm{~W}^{+} \mathrm{W}^{-}$pairs, $10^{6}$ Higgs bosons and $4 \cdot 10^{5}$ top-quark pairs per year. Such huge data samples combined with a $\mathscr{O}(100 \mathrm{keV})$ c.m. energy uncertainty will allow for Standard Model measurements with unparalleled precision and searches for new physics in regions not probed so far. The FCC-ee will be able to (i) indirectly discover new particles coupling to the Higgs and electroweak bosons up to scales $\Lambda \approx 7$ and $100 \mathrm{TeV}$; (ii) perform competitive SUSY tests at the loop level in regions beyond the $\mathrm{LHC}$ reach; and (iii) achieve the best potential in direct collider searches for dark matter and sterile neutrinos with masses below $60 \mathrm{GeV}$.
\end{abstract}

\section{Introduction}

Today, the understanding of particle physics is theoretically encoded in the Standard Model $(\mathrm{SM})$, a renormalizable quantum field theory -unifying quantum mechanics and special relativity - that describes the fundamental interactions (except gravity) via a local $\mathrm{SU}(3) \times$ $\mathrm{SU}(2) \times \mathrm{U}(1)$ gauge-symmetry group. The three gauge-symmetry terms give rise to the strong, weak and electromagnetic forces, while the particles fall into different representations of these symmetry groups. The SM Lagrangian (without neutrino masses) contains 19 free parameters to be determined experimentally: 3 gauge couplings, 9 Higgs-fermion Yukawa couplings, 3 mixing-angles, 2 Charge-Parity (CP) phases, and 2 Higgs boson couplings. Despite its tremendous success to accurately and consistently describe all phenomena observed at particle accelerators so far -including the recent experimental confirmation of the existence of its last missing piece, the Higgs boson- the theory is not complete and has several outstanding open questions to solve:

1. Dark matter (DM): The SM describes only $\sim 4 \%$ of the universe energy budget, the rest being in unknown DM (and dark energy) forms, pointing to the existence of new massive particles (such as e.g. SUSY partners, axions, heavy $\nu$ 's...).

2. Flavour problem: The huge dominance of matter over antimatter in the universe cannot be explained by the known SM sources of CP violation. More generally, the SM fails to explain the rationale behind the observed pattern of fermion families masses and flavour mixings.

3. Neutrino masses: The generation of non-zero neutrino masses, called for by the observation of their flavour oscillations, is beyond the SM (BSM) and requires new particles such as right-handed $\nu$ 's.

4. Hierarchy (or "fine tuning", "naturalness") problem: Quadratically-divergent virtual SM corrections affect the running of the Higgs boson mass between the widely separated electroweak and Planck scales, calling for new (e.g. supersymmetric) particles to stabilize such "untamed" quantum corrections.

5. Strong CP problem: The absence of CP-violation in QCD is naturally explained by postulating a (Peccei-Quinn) symmetry that gives rise to a new particle (axion).

6. Other fundamental SM issues include the current inability to explain dark energy, the cosmological constant, the origin of inflation, or gauge-gravity unification.

${ }^{a}$ E-mail: dde@cern.ch 
Many (or all) such fundamental questions may likely not be fully answered through the study of p-p collisions at the LHC. Despite their lower center-of-mass energies, $e^{+} e^{-}$colliders feature several advantages compared to their hadronic counterparts in terms of new physics studies: (i) direct model-independent searches for new particles coupling to $\mathrm{Z}^{*} / \gamma^{*}$ with masses up to $m \approx \sqrt{\mathrm{s}} / 2$; (ii) signals and backgrounds with very precisely known theoretical QCD and electroweak corrections with uncertainties (well) below 1\% in many cases; and (iii) very clean experimental environment with final states free of "holes" or "blind spots" typical of p-p searches involving new particles with difficult decay modes (e.g. in certain SUSY scenarios with soft final-states and/or without missing transverse energy). Thus, combined with high-luminosities, an $e^{+} e^{-}$collider can provide access to studies with permillevel precision $\delta X$, allowing one to place indirect constraints on BSM physics -appearing as virtual corrections to well-controlled processes- up to very-high energies $\Lambda \propto(1 \mathrm{TeV}) / \sqrt{\delta X}$. Plans exist to build future circular (FCC-ee [1], CEPC [2]) and/or linear (ILC [3], CLIC [4]) $e^{+} e^{-}$colliders (Fig. 1).

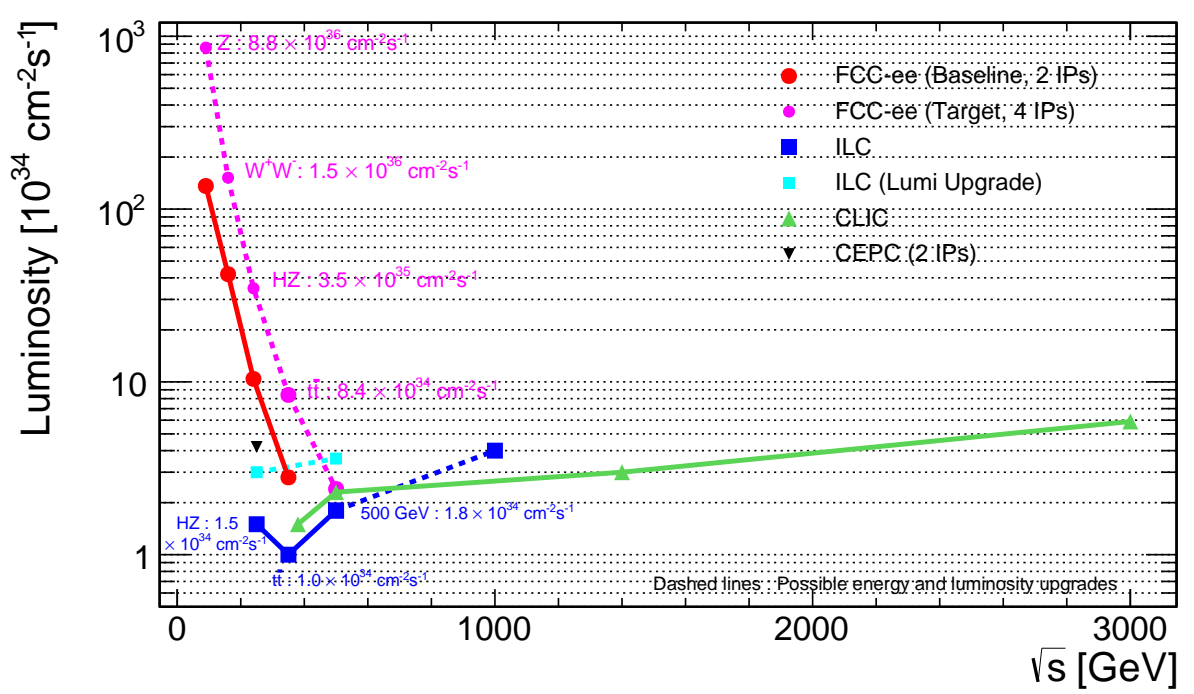

Figure 1: Target luminosities as a function of center-of-mass energy, in the range $\sqrt{\mathrm{s}} \approx 90-3000 \mathrm{GeV}$, for circular (FCC-ee, CEPC) and linear (ILC, CLIC) $e^{+} e^{-}$colliders currently under consideration.

The advantages of circular over linear $e^{+} e^{-}$machines are (i) much larger luminosity below $\sqrt{\mathrm{s}} \approx 400 \mathrm{GeV}$ (thanks to much higher collision rates, adding continuous top-up injection to compensate for luminosity burnoff); (ii) the possibility to have several interaction points (IPs); and (iii) a very precise measurement of the beam energy $E_{\text {beam }}$ through resonant transverse depolarization [5]. Linear $e^{+} e^{-}$colliders, on the other hand, feature (i) much larger $\sqrt{\mathrm{s}}$ reach (circular colliders are not competitive above $\sqrt{\mathrm{s}} \approx 400 \mathrm{GeV}$ due to synchroton radiation scaling as $\mathrm{E}_{\text {beam }}^{4} / R$ ), and (ii) easier longitudinal beam polarization. At the FCC (with a radius $\mathrm{R}=80-100 \mathrm{~km}$ ), $e^{+} e^{-}$collisions present clear advantages with respect to the older LEP $\left(\times 10^{4}\right.$ more bunches, and $\delta E_{\text {beam }}= \pm 0.1 \mathrm{MeV}$ compared to $\left.\pm 2 \mathrm{MeV}\right)$ and the ILC (crab-waist optics scheme, up to 4 IPs) yielding luminosities $\times\left(10^{4}-10\right)$ larger than both machines in the $\sqrt{\mathrm{s}}=90-350 \mathrm{GeV}$ range [6].

Table 1 lists the target FCC-ee luminosities and associated total number of events at each $\sqrt{\mathrm{s}}$. They have been obtained for the relevant cross sections including initial state radiation and smearings due to beam-energy spreads: $\sigma_{\mathrm{e}^{+} \mathrm{e}^{-} \rightarrow \mathrm{Z}}=43 \mathrm{nb}, \sigma_{\mathrm{e}^{+} \mathrm{e}^{-} \rightarrow \mathrm{H}}=0.29 \mathrm{fb}$, 
$\sigma_{\mathrm{e}^{+} \mathrm{e}^{-} \rightarrow \mathrm{W}^{+} \mathrm{W}^{-}}=4 \mathrm{pb}, \sigma_{\mathrm{e}^{+} \mathrm{e}^{-} \rightarrow \mathrm{HZ}}=200 \mathrm{fb}, \sigma_{\mathrm{e}^{+} \mathrm{e}^{-} \rightarrow \mathrm{t} \overline{\mathrm{t}}}=0.5 \mathrm{pb}$, and $\sigma_{\mathrm{e}^{+} \mathrm{e}^{-} \rightarrow \mathrm{VV} \rightarrow \mathrm{H}}=30 \mathrm{fb}$. The completion of the FCC-ee core physics program (described in the next sections) requires $\sim 10$ years of running.

\begin{tabular}{l|c|c|c|c|c|c}
\hline$\sqrt{\mathrm{s}}(\mathrm{GeV}):$ & $90(\mathrm{Z})$ & $125(\mathrm{eeH})$ & $160(\mathrm{WW})$ & $240(\mathrm{HZ})$ & $350(t \bar{t})$ & $350(\mathrm{WW} \rightarrow \mathrm{H})$ \\
\hline \hline $\mathscr{L} / \mathrm{IP}\left(\mathrm{cm}^{-2} \mathrm{~s}^{-1}\right)$ & $2.2 \cdot 10^{36}$ & $1.1 \cdot 10^{36}$ & $3.8 \cdot 10^{35}$ & $8.7 \cdot 10^{34}$ & $2.1 \cdot 10^{34}$ & $2.1 \cdot 10^{34}$ \\
$\mathscr{L}_{\text {int }}\left(\mathrm{ab}^{-1} / \mathrm{yr} / \mathrm{IP}\right)$ & 22 & 11 & 3.8 & 0.87 & 0.21 & 0.21 \\
Events/year $(4 \mathrm{IPs})$ & $3.7 \cdot 10^{12}$ & $1.2 \cdot 10^{4}$ & $6.1 \cdot 10^{7}$ & $7.0 \cdot 10^{5}$ & $4.2 \cdot 10^{5}$ & $2.5 \cdot 10^{4}$ \\
Years needed (4 IPs) & 2.5 & 1.5 & 1 & 3 & 0.5 & 3 \\
\hline
\end{tabular}

Table 1: Target luminosities, events/year, and years needed to complete the W, Z, H and top-quark programs at FCC-ee. [Note that $\mathscr{L}=10^{35} \mathrm{~cm}^{-2} \mathrm{~s}^{-1}$ corresponds to $\mathscr{L}_{\text {int }}=1 \mathrm{ab}^{-1} / \mathrm{yr}$ for 1 yr $=10^{7} \mathrm{~s}$ ].

These proceedings present succinctly the FCC-ee physics program. More detailed information can be found in [1], and in the dedicated physics FCC-ee (mini)workshops organized recently [7].

\section{Indirect constraints on BSM via high-precision electroweak and top physics}

Among the main goals of the FCC-ee is to collect multi-ab ${ }^{-1}$ at $\sqrt{\mathrm{s}} \approx 91 \mathrm{GeV}$ ( $\mathrm{Z}$ pole), $160 \mathrm{GeV}$ (WW threshold), and $350 \mathrm{GeV}$ ( $t \bar{t}$ threshold) in order to measure key properties of the $\mathrm{W}$ and $\mathrm{Z}$ bosons and the top-quark, as well as other fundamental SM parameters, with unprecedented precision. The combination of huge data samples available at each $\sqrt{\mathrm{s}}$ and the exquisite control of the c.m. energy (at the $\pm 100 \mathrm{keV}$ level) leading to very accurate energy threshold scans, allows the experimental precision of many SM parameters to be improved by a factor better than 25 with respect to the current state of the art (Table 2) [8]. Some FCC-ee experimental precision targets are $\pm 100 \mathrm{keV}$ for $m_{\mathrm{z}}, \pm 500 \mathrm{keV}$ for $m_{\mathrm{W}}, \pm 10 \mathrm{MeV}$ for $m_{\mathrm{t}}$, a relative statistical uncertainty of the order of $3 \cdot 10^{-5}$ for the QED $\alpha$ coupling (through $e^{+} e^{-} \rightarrow \mu^{+} \mu^{-}$forward-backward asymmetries above and below the $\mathrm{Z}$ peak) [9], one-permil for the QCD coupling $\alpha_{\mathrm{s}}$ (through hadronic Z and W decays) [10], and one-permil on the electroweak top couplings $F_{1 \mathrm{~V}, 2 \mathrm{~V}, 1 \mathrm{~A}}^{\gamma t, Z t}$ (through angular distributions in $\left.e^{+} e^{-} \rightarrow t \bar{t} \rightarrow \ell \nu q \bar{q} b \bar{b}\right)$ [11]. In many cases, the dominant uncertainty will be of theoretical origin, and developments in the calculations are needed in order to match the expected experimental uncertainty.

None of these measurements can be carried out at the LHC (or other $e^{+} e^{-}$machines) with such a level of precision. Physics beyond the SM can thereby be indirectly probed through loop corrections induced by possible new heavy particles [12]. Figure 2 shows limits on the W-mass vs. top-mass plane (left), and on the subset of leptonic dimension- 6 operators of a model-independent Effective Field Theory of the SM parametrizing possible new physics (right) [13]. Such measurements impose unrivaled constraints on new weaklycoupled physics. Whereas electroweak precision tests (EWPT) at LEP bound any BSM physics at scales $\Lambda \gtrsim 7 \mathrm{TeV}$, FCC-ee would reach up to $\Lambda \approx 100 \mathrm{TeV}$ for some operators.

\section{Indirect constraints on BSM via high-precision Higgs physics}

The Higgs sector of the SM can be probed with a unique precision with a high-luminosity lepton collider. In the range of FCC-ee energies, Higgs production peaks at $\sqrt{\mathrm{s}} \approx 240 \mathrm{GeV}$ dominated by Higgsstrahlung $\left(e^{+} e^{-} \rightarrow \mathrm{HZ}\right)$, with some sensitivity at $\sqrt{\mathrm{s}}=350 \mathrm{GeV}$ also 


\begin{tabular}{c|c|c|c|c|c}
\hline Observable & Measurement & Current precision & FCC-ee stat. & Possible syst. & Challenge \\
\hline \hline$m_{\mathrm{z}}(\mathrm{MeV})$ & Z lineshape & $91187.5 \pm 2.1$ & 0.005 & $<0.1$ & QED corr. \\
$\Gamma_{\mathrm{z}}(\mathrm{MeV})$ & Z lineshape & $2495.2 \pm 2.3$ & 0.008 & $<0.1$ & QED corr. \\
$R_{\ell}$ & Z peak & $20.767 \pm 0.025$ & 0.0001 & $<0.001$ & QED corr. \\
$R_{\mathrm{b}}$ & Z peak & $0.21629 \pm 0.00066$ & 0.000003 & $<0.00006$ & $g \rightarrow \mathrm{b} \overline{\mathrm{b}}$ \\
$N_{\nu}$ & $\mathrm{Z}$ peak & $2.984 \pm 0.008$ & 0.00004 & 0.004 & Lumi meas. \\
$N_{\nu}$ & $\mathrm{e}^{+} \mathrm{e}^{-} \rightarrow \gamma \mathrm{Z}($ inv. $)$ & $2.92 \pm 0.05$ & 0.0008 & $<0.001$ & - \\
$A_{\mathrm{FB}}^{\mu \mu}$ & $\mathrm{Z}$ peak & $0.0171 \pm 0.0010$ & 0.000004 & $<0.00001$ & $E_{\text {beam meas. }}$ \\
$\alpha_{\mathrm{s}}\left(m_{\mathrm{z}}\right)$ & $R_{\ell}, \sigma_{\text {had }}, \Gamma_{\mathrm{z}}$ & $0.1190 \pm 0.0025$ & 0.000001 & 0.00015 & New physics \\
$1 / \alpha_{\mathrm{QED}}\left(m_{\mathrm{Z}}\right)$ & $A_{\mathrm{FB}}^{\mu \mu}$ around Z peak & $128.952 \pm 0.014$ & 0.004 & 0.002 & EW corr. \\
\hline$m_{\mathrm{W}}(\mathrm{MeV})$ & $\mathrm{WW}$ threshold scan & $80385 \pm 15$ & 0.3 & $<1$ & QED corr. \\
$\alpha_{\mathrm{s}}\left(m_{\mathrm{W}}\right)$ & $\Gamma_{\mathrm{W}}, B_{\mathrm{had}}^{\mathrm{W}}$ & $B_{\mathrm{had}}^{\mathrm{W}}=67.41 \pm 0.27$ & 0.00018 & 0.00015 & CKM matrix \\
\hline$m_{\mathrm{t}}(\mathrm{MeV})$ & $t \bar{t}$ threshold scan & $173200 \pm 900$ & 10 & 10 & QCD \\
$\Gamma_{\mathrm{t}}(\mathrm{MeV})$ & $t \bar{t}$ threshold scan & $1410_{-150}^{+290}$ & 12 & $?$ & $\alpha_{\mathrm{s}}\left(m_{\mathrm{z}}\right)$ \\
$y_{\mathrm{t}}$ & $t \bar{t}$ threshold scan & $\mu=2.5 \pm 1.05$ & $13 \%$ & $?$ & $\alpha_{\mathrm{s}}\left(m_{\mathrm{z}}\right)$ \\
$F_{1 \mathrm{~V}, 2 \mathrm{~V}, 1 \mathrm{~A}}^{\gamma t, Z}$ & $\mathrm{~d} \sigma^{t \bar{t}} /$ dx dcos $(\theta)$ & $4 \%-20 \%(\mathrm{LHC}-14 \mathrm{TeV})$ & $(0.1-2.2) \%$ & $(0.01-100) \%$ & - \\
\hline
\end{tabular}

Table 2: Examples of achievable precisions in representative Z, W and top measurements at FCC-ee.
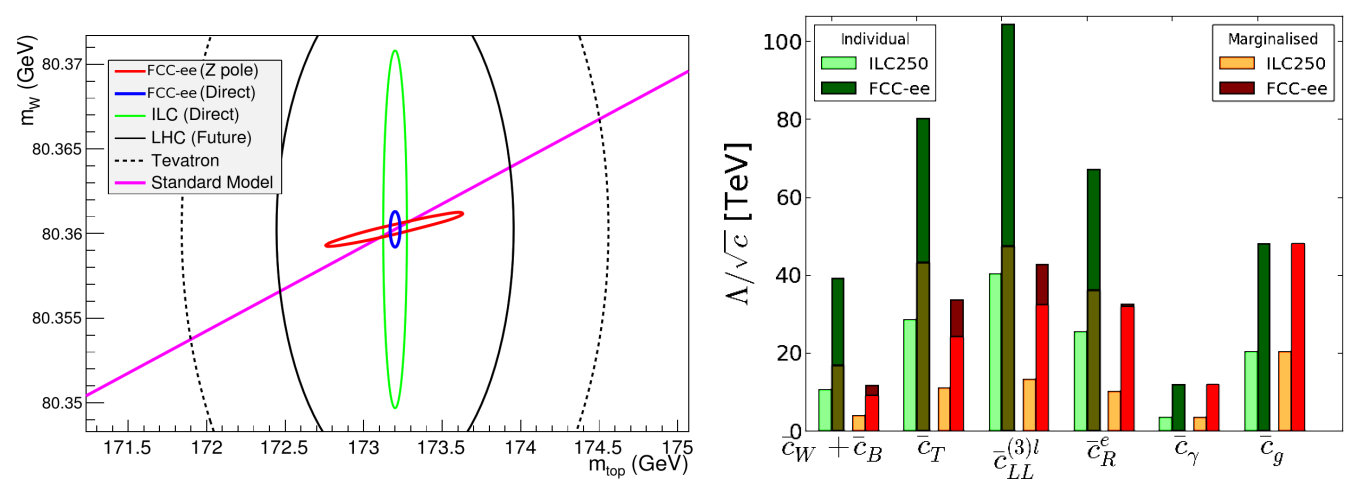

Figure 2: Left: Comparisons of $68 \%$ C.L. limits in the $m_{\mathrm{t}}-m_{\mathrm{W}}$ plane at FCC-ee and other colliders [1]. Right: $95 \%$ C.L. limits at FCC-ee(250 GeV) for leptonic operators sensitive to EWPT [13]. 
to vector-boson-fusion $\left(V V \rightarrow \mathrm{H} e^{+} e^{-}, \nu \nu\right)$ and the top Yukawa coupling $y_{\mathrm{t}}$ (via $e^{+} e^{-} \rightarrow t \bar{t}$ with a virtual Higgs exchanged among the top quarks). The target total number of Higgs produced at the FCC-ee (4 IPs combined, all years) amounts to 2.1 million at $240 \mathrm{GeV}$, 75000 in $V V \rightarrow \mathrm{H}$ at $350 \mathrm{GeV}$, and 19000 in s-channel $e^{+} e^{-} \rightarrow \mathrm{H}$ at $\sqrt{\mathrm{s}}=125 \mathrm{GeV}$ (Table 1). Unique Higgs physics topics are open to study with such large data samples:

- High-precision model-independent determination of the Higgs couplings, total width, and exotic and invisible decays (Fig. 3 right) [1].

- Higgs self-coupling through loop corrections in HZ production [14].

- First-generation fermion couplings: $(\mathrm{u}, \mathrm{d}, \mathrm{s})$ through exclusive decays $\mathrm{H} \rightarrow \mathrm{V} \gamma(\mathrm{V}=$ $\rho, \omega, \phi)[15]$, and electron Yukawa through resonant $e^{+} e^{-} \rightarrow \mathrm{H}$ at $\sqrt{\mathrm{s}}=m_{\mathrm{H}}[16]$.

The recoil mass method in $e^{+} e^{-} \rightarrow \mathrm{HZ}$ is unique to lepton colliders, and allows for an accurate tagging of Higgs events (Fig. 3, left) irrespective of their decay mode. It provides, in particular, a high-precision $( \pm 0.4 \%)$ measurement of $\sigma_{\mathrm{e}^{+} \mathrm{e}^{-} \rightarrow \mathrm{HZ}}$ and, therefore, of $g_{\mathrm{HZ}}^{2}$. From the measured value of $\sigma_{\mathrm{e}^{+} \mathrm{e}^{-} \rightarrow \mathrm{H}(\mathrm{XX}) \mathrm{Z}} \propto \Gamma_{\mathrm{H} \rightarrow \mathrm{XX}}$ and the different known decays fractions $\Gamma_{\mathrm{H} \rightarrow \mathrm{XX}}$, one can then obtain the total Higgs boson width with $\mathscr{O}(1 \%)$ uncertainty combining different final states. The $\mathrm{HZ}\left(\ell^{+} \ell^{-}\right)$final state can be used to directly measure the invisible decay width of the Higgs boson in events where its decay products escape undetected, by analyzing the distribution of the mass recoiling against the lepton pair. The Higgs boson invisible branching fraction can be measured with an absolute precision of $0.2 \%$. If not observed, a $0.5 \%$ upper limit (95\% C.L.) can be set on this branching ratio [1].
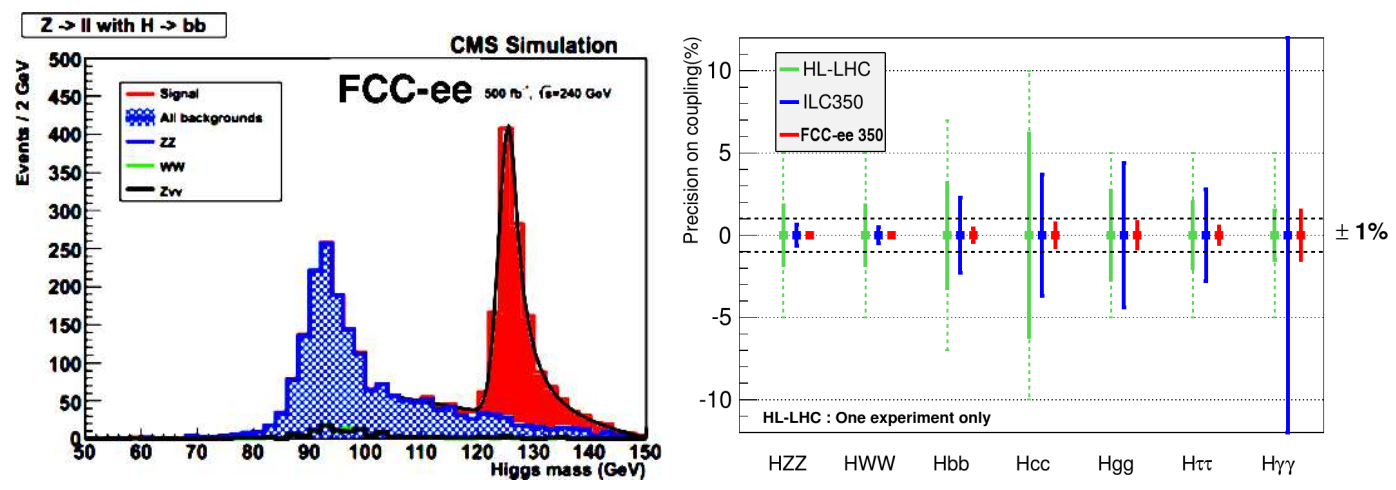

Figure 3: Left: Distribution of recoil mass against $\mathrm{Z} \rightarrow \ell \ell$ in the $e^{+} e^{-} \rightarrow \mathrm{HZ}$ process with $\mathrm{H} \rightarrow b \bar{b}$. Right: Comparison of expected relative uncertainties for the Higgs boson couplings at future facilities [1].

In addition, loop corrections to the Higgsstrahlung cross sections at different center-ofmass energies are sensitive to the Higgs self-coupling. The effect is tiny but visible at FCC-ee thanks to the extreme precision achievable on the $g_{\mathrm{HZ}}$ coupling. Indirect (model-dependent) limits on the trilinear $g_{\mathrm{HHH}}$ can be set with a $\mathscr{O}(70 \%)$ uncertainty, comparable to that expected at HL-LHC [14]. The large Higgs data samples available also open up the study of exotic (e.g. flavour-violating Higgs) and very rare SM decays. The Higgs couplings to firstand second-generation fermions, which may reveal new dynamics on the flavour structure of the SM [17], can be accessed via exclusive decays $\mathrm{H} \rightarrow \mathrm{V} \gamma$, for $V=\rho, \omega, \phi$, with sensitivity to the $\mathrm{u}, \mathrm{d}, \mathrm{s}$ quark Yukawas [15]. The $\mathrm{H} \rightarrow \rho \gamma$ channel appears the most promising with $\mathscr{O}(50)$ events expected. The low mass of the electron translates into a tiny $\mathrm{H} \rightarrow \mathrm{e}^{+} \mathrm{e}^{-}$branching ratio $\mathrm{BR}_{\mathrm{e}^{+} \mathrm{e}^{-}}=5 \cdot 10^{-9}$ which precludes any experimental observation of this decay mode and, thereby a determination of the electron Yukawa coupling. The resonant s-channel 
production, despite its small cross section [18], is not completely hopeless and preliminary studies indicate that could be observed at the $3 \sigma$-level with $90 \mathrm{ab}^{-1}$ at $\sqrt{\mathrm{s}}=125 \mathrm{GeV}$ with a c.m. energy spread commensurate with the Higgs boson width itself $(\approx 4 \mathrm{MeV}$, requiring beam monochromatization) [16].

In summary, the FCC-ee provides the smallest uncertainties for the measurements of Higgs boson couplings to gauge bosons and fermions (Fig. 3, right). Since any deviation $\delta g_{\mathrm{HXx}}$, relative to the SM value $g_{\mathrm{HXX}}^{\mathrm{SM}}$, can be approximately translated into BSM scale limits through the expression: $\Lambda \gtrsim(1 \mathrm{TeV}) / \sqrt{\left(\delta \mathrm{g}_{\mathrm{Hxx}} / \mathrm{g}_{\mathrm{HXX}}^{\mathrm{SM}}\right) / 5 \%}$. The expected $0.15 \%$ precision for the most precise coupling, $g_{\mathrm{HzZ}}$, would thus set competitive bounds, $\Lambda \gtrsim 7 \mathrm{TeV}$, on any new physics coupled to the scalar sector of the SM.

\section{Indirect constraints on supersymmetry}

Supersymmetry has many appealing features as a candidate framework for BSM physics, solving the naturalness problem (through light stop contributions to the running of the Higgs boson mass), stabilizing the electroweak vacuum, providing dark matter candidate(s) (the stable lightest SUSY particle, if R-parity is conserved), predicting grand unification of gauge interactions, and connecting to string theory where SUSY is preserved. Since stops and

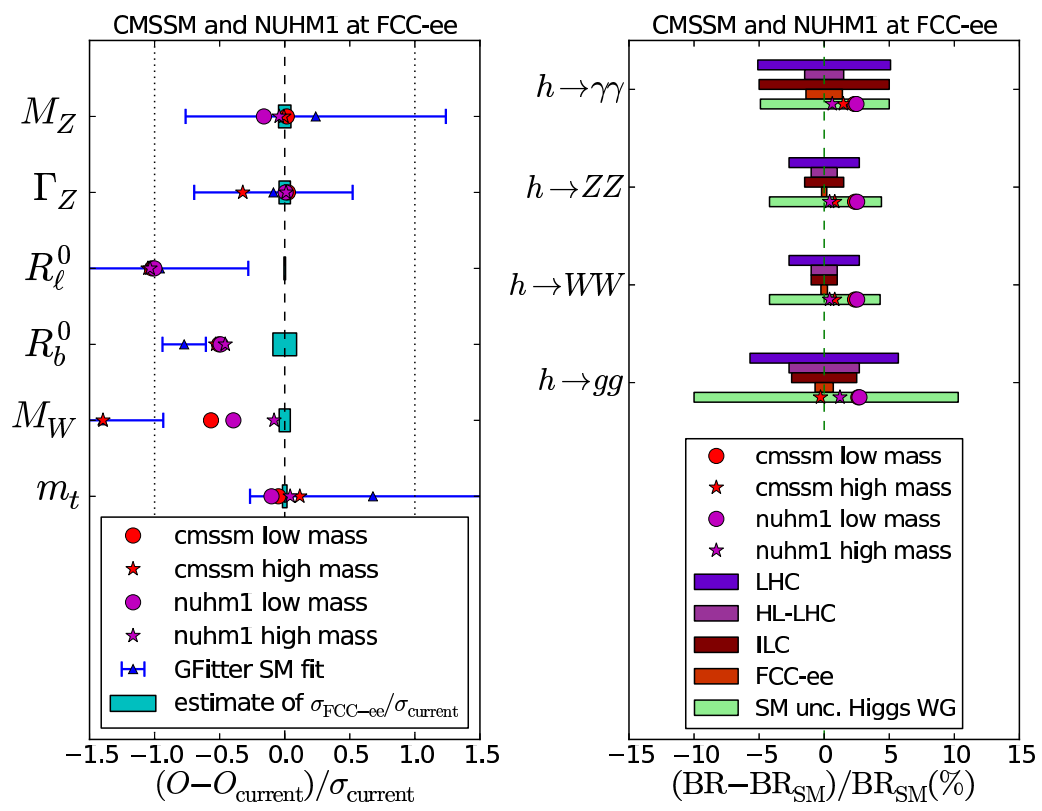

Figure 4: Comparison of the estimated precision in EWPT (left) and Higgs branching ratios (right) measurements: Current results (error bars, left; green boxes, right), low- and high-mass best-fit CMSSM and NUHM1 points (circles and stars) and prospects at future colliders (bars) [19].

higgsinos carry electroweak quantum numbers, $e^{+} e^{-}$colliders have better sensitivity to those than to coloured sparticles (squarks, gluinos). The precision electroweak and top (Section 2) and Higgs (Section 3) studies at the FCC-ee not only impose generic constraints on new physics scales at multi-TeV energies, but can also be interpreted in terms of sensitivity to broad classes of SUSY models (such as the Constrained MSSM, Non-Universal Higgs Masses, or natural SUSY) [19,20]. Figure 4 compares the precision on EWPT (left) and Higgs (right) observables at the LHC, ILC and FCC-ee with the deviations from their SM 
values expected for the low- and high-mass CMSSM and NUHM1 best-fit points [1, 19]. It is clear that the FCC-ee has the best ability to distinguish these models from the Standard Model. In particular, the high-mass CMSSM points in Fig. 4 provide an example of SUSY model which likely lies beyond the LHC reach, featuring narrow strips where stop-neutralino coannihilation is important, or focus-point strips at higher values of the ratio $m_{0} / m_{1 / 2}$. The EWPT and Higgs measurements at the FCC-ee would be able to probe both types of narrow parameter-space strips that extend to large sparticle masses, and indirectly determine CMSSM parameters also in such a pessimistic scenario [19].

\section{Direct constraints on BSM physics: dark matter and right-handed neutrinos}

The impact of the FCC-ee goes beyond indirect BSM studies and has also a strong discovery potential in direct searches of other key BSM extensions such as dark matter (DM) [21] and right-handed neutrinos [22], by exploiting the possibility to measure very rare decays of the $\mathrm{Z}$ and $\mathrm{H}$ bosons into such new particles. As a matter of fact, measurements of the invisible $\mathrm{Z}$ and $\mathrm{H}$ widths at the FCC-ee provide the best collider options to test DM lighter than $m_{\mathrm{Z}, \mathrm{H}} / 2$ that couples via SM mediators. Figure 5 (left) shows the limits in the plane (branching ratio, DM mass) for the decays $\mathrm{Z}, \mathrm{H} \rightarrow \mathrm{DM}$ DM. Similarly, Fig. 5 (right) shows the unrivaled sensitivity of searches for high-mass sterile neutrinos via decays $\mathrm{Z} \rightarrow \mathrm{N} \nu_{\mathrm{i}}$ (with $\mathrm{N} \rightarrow \mathrm{W}^{*} \ell, \mathrm{Z}^{*} \nu_{\mathrm{j}}$ ) as a function of their mass and mixing to light neutrinos (normal hierarchy) [22].
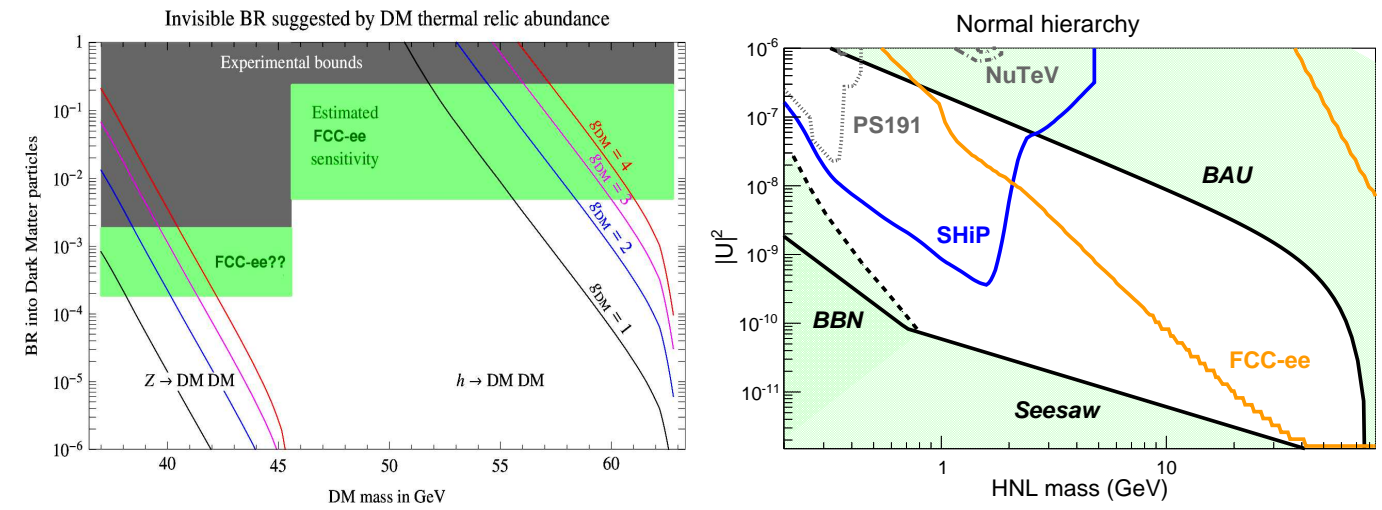

Figure 5: Regions of FCC-ee sensitivity for: (i) Rare Z and H decays into DM pairs in the $\mathrm{BR}_{\mathrm{Z}, \mathrm{H} \rightarrow \mathrm{DM} \mathrm{DM}}$ vs. $\mathrm{m}_{\mathrm{DM}}$ plane (left) [21], and (ii) sterile neutrinos as a function of their mass and mixing to light neutrinos (normal hierarchy) for $10^{13} \mathrm{Z}$ decays (right) [22].

\section{Summary}

The FCC-ee has a unique program of searches for new physics via high-precision studies of the W, Z, and Higgs bosons, and the top quark, with uncertainties at the permil level or below thanks to the huge luminosities $\mathscr{O}(1-100) \mathrm{ab}^{-1}$ (for 4 interaction points) and the exquisite control of the beam energy in the range $\sqrt{\mathrm{s}} \approx 90-350 \mathrm{GeV}$. By searching for tiny deviations with respect to the SM predictions in a rich set of measurements, BSM physics scales as large as $\Lambda \approx 7,100 \mathrm{TeV}$ for new particles coupling to the scalar and electroweak SM sectors respectively, can be indirectly probed. When interpreted in terms of broad classes of SUSY models, such precision electroweak and Higgs observables provide indirect tests of supersymmetry in regions which are beyond the LHC reach. Last but not least, the FCC-ee 
covers also direct BSM searches, through very rare and invisible Higgs and Z bosons decays which provide the best collider options to test dark matter and sterile neutrinos with masses up to $m_{\mathrm{DM}, \mathrm{HNL}} \approx 60 \mathrm{GeV}$.

\section{References}

[1] M. Bicer et al. [TLEP Design Study Working Group], JHEP 1401, 164 (2014); [arXiv:1308.6176 [hep-ex]].

[2] M. Ahmad et al. [CEPC-SPPC Study Group], IHEP-CEPC-DR-2015-01, IHEP-TH2015-01, HEP-EP-2015-01 (2015).

[3] T. Behnke et al. [ILC Collaboration], arXiv:1306.6327 [physics.acc-ph].

[4] L. Linssen, A. Miyamoto, M. Stanitzki and H. Weerts, doi:10.5170/CERN-2012-003; [arXiv:1202.5940 [physics.ins-det]].

[5] M. Koratzinos, A. Blondel, E. Gianfelice-Wendt and F. Zimmermann, arXiv:1506.00933 [physics.acc-ph].

[6] F. Zimmermann, 1st FCC annual workshop, Washington, March 2015; https://indico.cern.ch/event/340703/session/109/contribution/190/

[7] $1^{\text {st }}$ FCC-ee workshop on precision observables and radiative corrections, July 2015, https://indico.cern.ch/event/387296/

$1^{\text {st }}$ FCC-ee Higgs physics workshop, Sep. 2015, https://indico.cern.ch/event/401590/ Workshop on $\alpha_{\mathrm{s}}$ at FCC-ee, Oct. 2015, https://indico.cern.ch/e/alphas2015

FCC-ee precision physics workshop, Feb. 2016, https://indico.cern.ch/event/469561/

[8] R. Tenchini, arXiv:1412.2928 [hep-ex].

[9] P. Janot, JHEP 1602, 053 (2015); arXiv:1512.05544 [hep-ph].

[10] D. d'Enterria et al., arXiv:1512.05194 [hep-ph].

[11] P. Janot, JHEP 1504, 182 (2015); [arXiv:1503.01325 [hep-ph]].

[12] J. Fan, M. Reece and L. T. Wang, JHEP 1509, 196 (2015); [arXiv:1411.1054 [hep-ph]].

[13] J. Ellis and T. You, arXiv:1510.04561 [hep-ph].

[14] M. McCullough, Phys. Rev. D 90, 015001 (2014) [arXiv:1312.3322 [hep-ph]].

[15] A. L. Kagan, G. Perez, F. Petriello, Y. Soreq, S. Stoynev and J. Zupan, Phys. Rev. Lett. 114, 101802 (2015); [arXiv:1406.1722 [hep-ph]].

[16] D. d'Enterria, G.Wojcik, R.Aleksan, 8th FCC-ee Physics Workshop, LPNHE-Paris, Oct. 2014, https://indico.cern.ch/event/337673/session/6/contribution/20

[17] D. Ghosh, R. S. Gupta and G. Perez, arXiv:1508.01501 [hep-ph].

[18] S. Jadach and R. A. Kycia, Phys. Lett. B 75558 (2016); arXiv:1509.02406 [hep-ph].

[19] O. Buchmueller, M. Citron, J. Ellis, et al., Eur. Phys. J. C 75, 469 (2015); [arXiv:1505.04702 [hep-ph]].

[20] J. Fan, M. Reece and L. T. Wang, JHEP 1508, 152 (2015); [arXiv:1412.3107 [hep-ph]].

[21] A. De Simone, G. F. Giudice and A. Strumia, JHEP 1406, 081 (2014) [arXiv:1402.6287 [hep-ph]]; A. Strumia, 9th FCC-ee Physics Workshop, SNS-Pisa, Feb. 2015, http://www.sns.it/eventi/9th-fcc-ee-physics-workshop

[22] A. Blondel et al. [FCC-ee study Team Collaboration], arXiv:1411.5230 [hep-ex]. 